\title{
Prevalence, Modes of Management and Treatment outcomes among Patients with HIVIAIDS and Tuberculosis Co-infections in Dar es Salaam, 2005
}

\author{
Maro I, Mgonda Y.
}

\begin{abstract}
Objectives: To determine the prevalence, modes of management and treatment outcomes among patients with HIV/TB co-infection attending Dar-es-Salaam hospitals in 2005.

Methodology: Retrospective, Cross-sectional hospital based study was conducted between $12^{\text {th }}$ August to $23^{\text {rd }}$ September 2005. Data were collected among patients attending VCT clinics at Muhimbili National Hospital and Mnazi Mmoja Hospital. The study included medical records of patients aged $\geq 8$ years.
\end{abstract}

Results: HIV infected individuals had a higher (20.2\%) prevalence of Tuberculosis compared to those who were negative for HIV (3.2\%).

The most commonly used diagnostic methods included radiological tests, sputum smears and tuberculin skin tests. Sputum cultures were hardly done.

Chest radiography was found to be atypical in most of the patients who were positive for HIV 17 (32.7\%). Sputum smears were found to be negative among $10(19.2 \%)$ of the patients who were positive for TB through other tests.

Treatment for Tuberculosis and AIDS depends on the CD4 levels and that TB was treated first before HAART in the case where the CD4 were < 200 and anti-TB was administered for a short while until patients were stable. Most (90.9\%) of patients with CD4 $<50$ were not on anti-TB therapy at the time of the study but they had a history of being treated for $T B$ earlier before the start of ARVs. No side effects from the drug therapy were observed in all patients involved in this study. None among the study population died in the period of the study.

Conclusion: In this study, TB infection was found to be significantly higher among patients who are HIV positive than not. Chest radiography was found to be atypical in most of the HIV infected patients.

Recommendations: All HIV infected individuals should be tested for Tuberculosis prior to the initiation of ART in countries (like Tanzania) where tuberculosis is endemic. Several tests including chest $x$-ray and sputum cultures should be done to all individuals. This will result in early detection and treatment, and thus reduction of spread of disease.

Correspondence to: Dr. Isaac Marso. Tel: +255-713-311-300, E-mail: isaacmarso@yahoo.com

\section{Introduction}

There is a growing number of individuals with HIV/Tuberculosis co-infections, posing several challenges in early recognition, diagnosis, prevention, prophylaxis and treatment of tuberculosis.

Diagnosis of TB in HIV/AIDS patients can be difficult as most of the potentially used diagnostic tests are usually negative or atypical. This poses a diagnostic challenge that is yet to be resolved. On the other hand treatment of TB in patients with symptomatic HIV infection can be a challenge mainly because of interactions among drugs used for TB. There is no best approach to this so far. Likewise the prevalence of adverse reactions to anti-TB medications is not clearly known in Tanzania. The overall survival rate for patients with TB-HIV co-infection is also unknown.

\section{Methodology}

This retrospective, cross-sectional study was hospital based and conducted for a period of 6 weeks, from $12^{\text {th }}$ August to $23^{\text {rd }}$ September 2005. The aim was to determine the prevalence, modes of management, diagnostic modalities and treatment outcomes in patients with HIV/AIDS and Tuberculosis co-infection. The study involved patients attending Dar es salaam hospitals in the year 2004. Data collected involved reviewing files of patients who were attending VCT clinics at Muhimbili National Hospital and mnazi mmoja hospital between $1^{\text {st }}$ November 2002 and $31^{\text {st }}$ January 2003. The study involved medical records regarding patients aged 8 years and above attending the selected VCT clinics in Dar es salaam. 
Results

Our study recruited 1318 patients. It also revealed that HIV infected patients had a significantly higher prevalence $(20.2 \%)$ of tuberculosis compared to those individuals who were negative for HIV (3.2\%).

The most commonly used diagnostic methods included chest radiographs, sputum smears and tuberculin skin tests. Sputum cultures were also done in these clinics

Chest radiographs were found to be atypical in most of the patients who were positive for HIV $(32.7 \%)$ Sputum smears were found to be negative in $19.2 \%$ of the patients who were found to be positive for TB through other tests.

The study also found that treatment for Tuberculosis and AIDS depends on the CD4 levels and that TB was treated first before HAART in the case where CD4 were $<200$. Anti-TB was administered for a short while until patients were stable. Almost all $(90.9 \%)$ patients with CD4 $<50$ were not using anti-TB therapy at the time of the study but they had a history of being treated for TB earlier before the start of ARVs. No side effects from drug therapy were observed in all the patients involved in this study.

Figure no.1: The pattern of HIV/AIDS and Tuberculosis co-infection in Dar es Salaam

\begin{tabular}{|l|l|l|l|l|l|l|}
\hline \multirow{2}{*}{ HIV } & \multicolumn{3}{c}{ TOSITIVE } & \multicolumn{2}{c|}{ NEGATIVE } & \multicolumn{2}{c|}{ TOTAL } \\
& Frequency & $\%$ & Frequency & $\%$ & Frequency & $\%$ \\
\hline POSITIVE & 70 & 20.2 & 277 & 79.8 & 347 & 26.3 \\
\hline NEGATIVE & 31 & 3.2 & 940 & 96.8 & 971 & 73.1 \\
\hline TOTAL & 101 & 7.7 & 1217 & 92.3 & 1318 & 100 \\
\hline
\end{tabular}

From the figure no.1 (above), the prevalence rate of HIV/TB co-infection in this study population was found to be $20.2 \%$. The table also shows that the prevalence of HIV infection was $26.3 \%$ while that of TB was $7.7 \%$.

Figure no.2: Prevalence of HIV-infected patients who were on anti-TB regimens

\begin{tabular}{|l|l|l|l|l|l|l|}
\hline HIV & ON TREATMENT & NOT ON TREATMENT & \multicolumn{2}{c|}{ TOTAL } \\
\hline & No & $\%$ & No & $\%$ & No & $\%$ \\
\hline POSITIVE & 26 & 37.1 & 44 & 62.9 & 70 & 69.3 \\
\hline NEGATIVE & 12 & 38.7 & 19 & 61.3 & 31 & 30.7 \\
\hline TOTAL & 38 & 37.6 & 63 & 62.4 & 101 & 100.0 \\
\hline
\end{tabular}

Figure no. 3: PROPORTION OF PATIENTS WHO WERE RECEIVING TB REGIMEN AND THEIR CD4 LEVELS

\begin{tabular}{|l|l|l|l|l|l|l|}
\hline CD4 & \multicolumn{2}{l}{ ON TREATMENT } & \multicolumn{2}{l|}{ NO TREATMENT } & \multicolumn{2}{l|}{ TOTAL } \\
\cline { 2 - 7 } & No & $\%$ & No & $\%$ & No & $\%$ \\
\hline$<50$ & 4 & 9.1 & 40 & 90.9 & 44 & 62.9 \\
\hline $50-200$ & 8 & 66.7 & 4 & 33.3 & 12 & 17.1 \\
\hline$>200$ & 14 & 100.0 & 0 & 0 & 14 & 20.0 \\
\hline TOTAL & 26 & 37.1 & 44 & 62.9 & 70 & 100.0 \\
\hline
\end{tabular}

Among 127 sputum samples and 13 lymph nodes samples studied, 35 sputum and 5 lymph nodes samples grew significant
Mycobacterium tuberculosis organisms that were subsequently sub-cultured in media containing the specific anti-tuberculosis 
drugs. It was found that the isolated $M$. tuberculosis was sensitive to the tested anti-tuberculosis drugs in all the subcultures, signifying absence of primary drug resistance with these isolates.

\section{Discussion}

The HIV and tuberculosis epidemics are fueling each other, creating a public health crisis of enormous proportions. ${ }^{1}$ HIV destroys the body's immune system and makes the individual prone to opportunistic infections (e.g. TB). Patients with HIV/AIDS can develop active tuberculosis either as a result of re-activation of their chronic latent TB or by acquiring new infections from the environment. ${ }^{2}$

In this study, it was found that HIV infected individuals had a higher (20.2\%) prevalence of TB compared to those individuals who were negative for HIV (3.2\%). This study results were found to be similar to findings from earlier study done in Tanzania that revealed among patients with sputum positive results for Pulmonary TB, $35.8 \%$ were seropositive for $\mathrm{HIV}^{3}$. The study is also consistent to findings from the Dominican republic (a tropical country that is endemic for TB) which found the prevalence rates of $15 \%$ and $5 \%$ respectively ${ }^{4}$. However, the study shows a discrepancy from studies of other East African countries as evidence from studies done in Uganda and Kenya reveals ${ }^{5}$. One study done in Uganda revealed that only $2.6 \%$ of individuals reported to have TB-HIV co-infection. Another study done in Kenya revealed that $19 \%$ of HIV positive individuals had tuberculosis also.

From the study it was also found that many of the diagnostic modalities have shortcomings in diagnosing TB associated with HIV.

Chest radiography was found to be atypical in most patients who were positive for HIV $17(32.7 \%)$. This is in accordance with the study done in Tanzania that noted people with HIV infections to have atypical chest radiographs $^{3}$. Typical reactivation of tuberculosis on chest $\mathrm{x}$-rays was found in $51 \%$ of the HIV positive Tuberculosis patients ${ }^{3}$.
For many TB centres in Dar es Salaam, sputum smear microscopy remains the most feasible and practical technique for diagnosis of Tuberculosis. Patients suspected of having pulmonary tuberculosis submit three sputum specimens obtained on 3 consecutive days for examination of AFB. With high prevalence of HIV infection, the sensitivity of smear microscopy can decrease in HIV infected individuals. Studies have shown that AFB results were negative in $19.2 \%$ of patients who were found to be positive for TB through other tests. This was found to be in support with a study that revealed sensitivity of smear microscopy, which is about $70 \%$ among HIV seronegative individuals, can decrease in HIV infected individuals to about $31 \%$ depending on the stage of HIV disease ${ }^{4}$.

Cultures are golden standard in the diagnosis of TB. This technique is more sensitive than smear microscopy but it is very expensive, time consuming and requires extensive human and material resources. It is for this reason; the test is not routinely done in most of Dar es Salaam clinics.

It was observed that $90.9 \%$ of patients who had CD4 counts $>50$ were not on Tuberculosis regimen (DOTS). This is because some were on short period of AntiTuberculosis regimen before it was stopped for them to start HAART regimen. It was observed that $100 \%$ of patients with CD4 $>200$ were on DOTS regimens without HAART.

The study revealed that tuberculosis was treated first before initiation of HAART for at least a short while until the patient was stabilized. This is in accordance to the WHO guidelines that suggest that TB treatment must be given urgently in cases of severe immunocompromised HIV infection candidates $^{5}$.

The urgency of HIV treatment depends on predictors of HIV disease progression (HIV RNA level, CD4 cell count, prior HIV-related diagnoses).

Options are either to start HAART and Tuberculosis treatment together or starting HAART after acute rifampicin containing phase eg. 2 months or start HAART after the end of TB treatment. 
Now what if Tuberculosis develops while one is on HAART?

- Adapt HAART as necessary to avoid drug interactions.

- HAART initiation is rarely a vital emergency

HAART AND TB treatment is as follows:

When the patient is positive for TB and the cd4 count is less than $50(<50)$ or patient presents with extra pulmonary $\mathrm{Tb}$ then $\mathrm{Tb}$ therapy is started and as soon as the dose is tolerated then HAART commences.

When the patient is positive for TB and the CD4 count is between 50 and 200 then Tb regimen is started for 2 months after which HAART is then started.

When a patient is positive and presents with the CD4 counts of more than 200 then it is advised to treat for $\mathrm{Tb}$ and at the same time monitoring the CD4 counts so as to start ARV when CD4 fall below 200.

From the study it was observed that no resistance or side effects were observed. This was different from most of the studies where most of the time side effects are observed as seen in studies that were done in San fransisco where by, $18-26 \%$ of HIVseropositive patients with tuberculosis underwent a change in therapy because of adverse drug reactions ${ }^{5}$. Rifampin was the commonly implicated drug, producing an adverse reaction in $12 \%$ of the patients ${ }^{5}$. In the Democratic Republic of Congo (then Zaire), $11 \%$ of the seropositive cases developed a rash, but treatment was not interrupted. ${ }^{5}$

However this may not reflect what is in the general population taking into account of the small sample size. Similar findings were also found in another study done in 1993 in rural Iringa that found all culture positive samples samples $13.2 \%$ were sensitive to the tested anti-tuberculosis medications.

Low levels of resistance have been reported in Tanzania whereby it was found that about $90 \%$ of all newly diagnosed sputum smear positive cases are sensitive to streptomycin, isoniazid and thiacetazone ${ }^{3}$. Only in $8 \%$ were the bacilli resistant to isoniazid, In about $1-2 \%$ to streptomycin and in a further $1-2 \%$ to isoniazid and streptomycin while the resistance to rifampicin is very low $(1 \%)^{3}$.

No mortality were observed in the whole course of the study. This is in opposition to several studies which revealed that there are usually high mortality in HIV -Tb coinfected patients of up to $35 \%$.

\section{REFERENCES}

1. www.unaids.org/bangkok 2004

2. Small PM. Tuberculosis research-Balancing the portfolio. JAMA. 1996. 276:1512.

3. Noronha D, Pallangyo KJ. Radiological features in patients with infected Human Immnodeficiency Virus. East African Medical with Jornal 1991; 68: 201-5.

4. Chaisson RE et al; Am Rev Respir Dis 1987; 136:570-574.

5. Corbett EL, Watt CJ et al; Arch Intern Med. 2003; 163:1009-21

6. AIDS Control and Prevention (AIDSCAP), UNAIDS. Final Report, 1996

7. Manual of the NTLP in Tanzania. Fourth edition.2003 